\title{
Perturbation theorems of Miyadera type for locally Lipschitz continuous integrated semigroups
}

\author{
by \\ NAOKI TANAKA (Okayama)
}

\begin{abstract}
A class of perturbing operators for locally Lipschitz continuous integrated semigroups is introduced according to the idea of Miyadera. The paper gives perturbation theorems of Miyadera type for such integrated semigroups.
\end{abstract}

1. Introduction. This paper is devoted to perturbation theorems of Miyadera type for locally Lipschitz continuous integrated semigroups on a Banach space $X$ with norm $\|\cdot\|$.

The study of abstract Cauchy problems for non-densely defined operators satisfying the Hille-Yosida conditions was initiated by Da Prato and Sinestrari [3], and it has recently been done by using the theory of locally Lipschitz continuous integrated semigroups. It is known [5, Proposition 3.3] that bounded perturbations preserve the property of being a generator for such integrated semigroups. This is an extension of a perturbation theorem for semigroups of class $\left(C_{0}\right)$ due to Phillips.

Miyadera [7] introduced a class of perturbing operators for semigroups of class $\left(C_{0}\right)$ and obtained interesting perturbation theorems for such semigroups by using a convergence theorem. We are interested in obtaining a theorem unifying the above-mentioned two results, and we discuss the perturbation problem for locally Lipschitz continuous integrated semigroups.

We recall the definition of locally Lipschitz continuous integrated semigroups. Let $B(X)$ be the set of all bounded linear operators on $X$. A family $\{U(t) ; t \geq 0\}$ in $B(X)$ is called a locally Lipschitz continuous integrated semigroup on $X$ if it satisfies the following conditions:

2000 Mathematics Subject Classification: Primary 47D62; Secondary 47A55.

This research was partially supported by the Grant-in-Aid for Scientific Research (C)(2) No. 14540175, Japan Society for the Promotion of Science. 
(U1) For each $\tau>0$ there exists $L_{\tau}>0$ such that

$$
\|U(t)-U(s)\| \leq L_{\tau}|t-s| \quad \text { for } t, s \in[0, \tau] .
$$

$$
U(t) U(s) x=\int_{s}^{t+s} U(\tau) x d \tau-\int_{0}^{t} U(\tau) x d \tau \text { for } x \in X \text { and } t, s \geq 0 \text {. }
$$
If $U(t) x=0$ for all $t>0$ then $x=0$.

It is known [5, Corollary 2.3] that every locally Lipschitz continuous integrated semigroup $\{U(t) ; t \geq 0\}$ on $X$ is exponentially bounded in the sense that $\|U(t)\| \leq M e^{\omega t}$ for $t \geq 0$. In this case, there exists a unique closed linear operator $A$ in $X$ such that $(\omega, \infty)$ is contained in the resolvent set $\varrho(A)$ of $A$ and

$$
(\lambda I-A)^{-1} x=\int_{0}^{\infty} \lambda e^{-\lambda t} U(t) x d t
$$

for $x \in X$ and $\lambda>\omega$. The operator $A$ is called the generator of $\{U(t) ; t \geq 0\}$ on $X$ (see Arendt [1]). The following characterization of generators of locally Lipschitz continuous integrated semigroups was obtained in $[5$, Theorem 2.4]. A linear operator $A$ is the generator of a locally Lipschitz continuous integrated semigroup on $X$ if and only if there exist $M>0$ and $\omega \geq 0$ such that $(\omega, \infty) \subset \varrho(A)$ and

$$
\left\|(\lambda I-A)^{-m}\right\| \leq M(\lambda-\omega)^{-m} \text { for } m \geq 1 \text { and } \lambda>\omega .
$$

A simple example of a locally Lipschitz continuous integrated semigroup is the family $\{U(t) ; t \geq 0\}$ defined by

$$
U(t) x=\int_{0}^{t} T(s) x d s
$$

for $x \in X$ and $t \geq 0$, where $\{T(t) ; t \geq 0\}$ is a semigroup of class $\left(C_{0}\right)$ on $X$ generated by $A$. In this case, the generator of $\{U(t) ; t \geq 0\}$ is equal to $A$.

Acknowledgements. The author wishes to express his gratitude to the referee for several helpful suggestions.

2. A class of perturbing operators. According to the idea of Miyadera [7], we discuss the perturbation problem for locally Lipschitz continuous integrated semigroups by using a convergence theorem for such integrated semigroups. For this purpose, we introduce a class of perturbing operators as follows.

Let $A$ be the generator of a locally Lipschitz continuous integrated semigroup $\{U(t) ; t \geq 0\}$ on $X$. A linear operator $B$ is said to belong to the class $\mathfrak{P}(A)$ if there exists a sequence $\left\{B_{n}\right\}$ in $B(X)$ such that

$$
D(A) \subset D(B) \text { and } \lim _{n \rightarrow \infty} B_{n} x=B x \text { for every } x \in D(A),
$$

where $\operatorname{TV}(f ;[0,1])$ denotes the total variation of $f$ on $[0,1]$. 
We give two subclasses of $\mathfrak{P}(A)$.

Proposition 2.1. (a) Let $A$ be the generator of a semigroup $\{T(t) ; t \geq 0\}$ of class $\left(C_{0}\right)$ on $X$. If a linear operator $B$ in $X$ satisfies the two conditions

(a-i) $D(A) \subset D(B)$ and $B\left(\lambda_{0} I-A\right)^{-1} \in B(X)$ for some $\lambda_{0}$,

(a-ii) there exists $K>0$ such that

$$
\int_{0}^{1}\|B T(t) x\| d t \leq K\|x\| \quad \text { for } x \in D(A),
$$

then $B \in \mathfrak{P}(A)$.

(b) Let $A$ be the generator of a locally Lipschitz continuous integrated semigroup $\{U(t) ; t \geq 0\}$ on $X$. If a closed linear operator $B$ in $X$ satisfies the three conditions

(b-i) $D(B) \supset \bigcup_{t \geq 0} U(t)(X)$,

(b-ii) for each $x \in X, B U(\cdot) x:[0, \infty) \rightarrow X$ is continuous,

(b-iii) there exists $K>0$ such that

$$
\operatorname{TV}(B U(\cdot) x ;[0,1]) \leq K\|x\| \quad \text { for } x \in X,
$$

then $B \in \mathfrak{P}(A)$. In particular, if $B \in B(\overline{D(A)}, X)$ then $B \in \mathfrak{P}(A)$.

REMARK 2.1. The perturbation theory for semigroups of class $\left(C_{0}\right)$ was initiated and a class of perturbing operators ([4, Section 13.4, Definition 13.3.5]) was introduced by Phillips. The class of linear operators $B$ satisfying (a-i) and (a-ii) is the class of perturbing operators introduced by Miyadera [7]. It was proved in [7, Section 4] that this class properly includes the above-mentioned class of perturbing operators due to Phillips.

Proof of Proposition 2.1. To prove (a), we first notice that the operator $A$ is the generator of the locally Lipschitz continuous integrated semigroup $\{U(t) ; t \geq 0\}$ defined by (1.3). Consider $B_{n} \in B(X)$ defined by $B_{n}=$ $B(I-(1 / n) A)^{-1}$ for sufficiently large $n$. This definition is unambiguous because of condition (a-i) and the resolvent identity. Since

$$
\begin{aligned}
B(I-(1 / n) A)^{-1}\left(\lambda_{0} I-A\right)^{-1} x & =B\left(\lambda_{0} I-A\right)^{-1}(I-(1 / n) A)^{-1} x \\
& \rightarrow B\left(\lambda_{0} I-A\right)^{-1} x
\end{aligned}
$$

for every $x \in X$, we see that condition (P1) is satisfied. To check condition (P2), let $0=t_{0}<t_{1}<\ldots<t_{N}=1$ and $x \in X$. By (1.3) we have

$$
\sum_{i=1}^{N}\left\|B_{n} U\left(t_{i}\right) x-B_{n} U\left(t_{i-1}\right) x\right\| \leq \sum_{i=1}^{N} \int_{t_{i-1}}^{t_{i}}\left\|B T(s)(I-(1 / n) A)^{-1} x\right\| d s,
$$

so that $\operatorname{TV}\left(B_{n} U(\cdot) x ;[0,1]\right) \leq K\left\|(I-(1 / n) A)^{-1}\right\|\|x\|$ by condition (a-ii). This means that condition (P2) is satisfied. 
To prove (b), we need (ii-2) of Lemma 2.2 which will be proved below. By (ii-2) there exist $K_{0}>0$ and $\beta \geq 0$ such that $\|B U(t) x\| \leq K_{0} e^{\beta t}\|x\|$ for $x \in X$ and $t \geq 0$. This fact together with (1.1) implies that $D(A) \subset D(B)$ by the closedness of $B$, so that $B_{n}=B(I-(1 / n) A)^{-1} \in B(X)$ is well defined for sufficiently large $n$. Since $U(t)$ commutes with the resolvent of $A$ and $\left\|(I-(1 / n) A)^{-1}\right\| \leq M(1-\omega / n)^{-1}$ for some $M \geq 1$ and $\omega \geq 1$ (by (1.2)), condition (P2) is a direct consequence of (b-iii). By (1.1) and property (U2) we find

$$
\begin{aligned}
B_{n} U(t) x & =n^{2} \int_{0}^{\infty} e^{-n \tau} B U(\tau) U(t) x d \tau \\
& =n \int_{0}^{\infty} e^{-n \tau} B(U(\tau+t) x-U(\tau) x) d \tau
\end{aligned}
$$

for $x \in X$ and sufficiently large $n$, where we have used integration by parts to obtain the last equality. The right-hand side of the above equality is equal to $\int_{0}^{\infty} e^{-s} B(u(s / n+t) x-U(s / n) x) d s$, which tends to $B U(t) x$ as $n \rightarrow \infty$, by condition (b-ii). Notice that $\left\|B_{n} U(t) x\right\| \leq M K_{0} e^{\beta t}(1-\omega / n)^{-1}\|x\|$ for $x \in X$ and $n>\omega$. Condition (P1) follows readily from the above fact and (1.1), with the help of Lebesgue's convergence theorem.

Finally, let $B \in B(\overline{D(A)}, X)$ and $\|B x\| \leq M_{B}\|x\|$ for $x \in \overline{D(A)}$. Since $U(t)(X) \subset \overline{D(A)}$ for $t \geq 0$ (by the inversion formula [4, Theorem 6.3.5]), we see that conditions (b-i) and (b-ii) are satisfied. To check condition (b-iii), let $0=t_{0}<t_{1}<\ldots<t_{N}=1$. Then, by property (U1),

$$
\begin{aligned}
\left\|B U\left(t_{i}\right) x-B U\left(t_{i-1}\right) x\right\| & \leq M_{B}\left\|U\left(t_{i}\right) x-U\left(t_{i-1}\right) x\right\| \\
& \leq M_{B} L_{1}\left(t_{i}-t_{i-1}\right)\|x\| \quad \text { for } i=1, \ldots, N,
\end{aligned}
$$

so that $\operatorname{TV}(B U(\cdot) x ;[0,1]) \leq M_{B} L_{1}\|x\|$. It follows that $B \in \mathfrak{P}(A)$.

The following lemma will play an essential role in later arguments.

Let $A$ be the generator of a locally Lipschitz continuous integrated semigroup $\{U(t) ; t \geq 0\}$ on $X$. Then there exist $M \geq 1$ and $\omega \geq 0$ such that

$$
\|U(t+h)-U(t)\| \leq M e^{\omega(t+h)} h \quad \text { for } t, h \geq 0 .
$$

This fact is obtained by combining [5, Theorem 2.4] and [9, Theorem 3.1].

Lemma 2.2. (i) If a sequence $\left\{B_{n}\right\}$ in $B(X)$ satisfies condition (P2), then there exists $K>0$ such that

$$
\operatorname{TV}\left(B_{n} U(\cdot) x ;[0,1]\right) \leq K\|x\| \quad \text { for } x \in X \text { and } n \geq 1 .
$$

(ii) Let $B$ be a closed linear operator in $X$ satisfying conditions (b-i) through (b-iii). Let $\lambda>\omega$ and $0 \leq a=t_{0}<t_{1}<\ldots<t_{N}=b$. Then: 
(ii-1) For $x \in X$ we have

$$
\begin{aligned}
\sum_{l=1}^{N} e^{-\lambda t_{l}} \| & B U\left(t_{l}\right) x-B U\left(t_{l-1}\right) x \| \\
\leq & \begin{cases}\left(K+M K e^{-(\lambda-\omega)}\left(1-e^{-(\lambda-\omega)}\right)^{-1}\right)\|x\| & \text { for } 0 \leq a<1, \\
M K e^{-(\lambda-\omega)[a]}\left(1-e^{-(\lambda-\omega)}\right)^{-1}\|x\| & \text { for } a \geq 1,\end{cases}
\end{aligned}
$$

where $[a]$ denotes the integer part of $a$.

(ii-2) There exist $K_{0}>0$ and $\beta \geq 0$ such that $\|B U(t) x\| \leq K_{0} e^{\beta t}\|x\|$ for $x \in X$ and $t \geq 0$.

Proof. The proof is based on the idea of Miyadera [7, Lemma 1], but more delicate arguments are needed here. The functional $p$ defined by $p(x)=$ $\sup _{n \geq 1} \operatorname{TV}\left(B_{n} U(\cdot) x ;[0,1]\right)$ for $x \in X$ is a lower semicontinuous seminorm on $X$. If we set $X_{n}=\{x \in X ; p(x) \leq n\}$ for each $n \geq 1$, then each $X_{n}$ is closed in $X$ by the lower semicontinuity of $p$, and the union of all sets $X_{n}$ is equal to $X$. The Baire category theorem asserts that at least one $X_{n_{0}}$ contains an open ball with center $x_{0}$ and radius $R$. Since $p$ is a seminorm on $X$, we have $p(x) \leq(2\|x\| / R)\left\{p\left(x_{0}+R x /(2\|x\|)\right)+p\left(x_{0}\right)\right\} \leq\left(4 n_{0} / R\right)\|x\|$ for all $x \in X$ with $x \neq 0$, whence (i) follows.

To prove (ii), let $P=\left\{[a]=\tau_{0}<\tau_{1}<\ldots\right\}$ be the partition of $[[a], \infty)$ which is constructed by appending the points $\{k\}_{k=[a]}^{\infty}$ to the sequence $\left\{t_{i}\right\}_{i=0}^{N}$. By the construction of $P$, for each integer $k \geq[a]$ there exists an integer $i(k)$ such that $\tau_{i(k)}=k$. Let $x \in X$. Then

$$
\begin{aligned}
\sum_{l=1}^{N} e^{-\lambda t_{l}}\left\|B U\left(t_{l}\right) x-B U\left(t_{l-1}\right) x\right\| \leq \sum_{j=1}^{\infty} e^{-\lambda \tau_{j}}\left\|B U\left(\tau_{j}\right) x-B U\left(\tau_{j-1}\right) x\right\| \\
=\sum_{k=[a]+1}^{\infty} \sum_{j=i(k-1)+1}^{i(k)} e^{-\lambda \tau_{j}}\left\|B U\left(\tau_{j}\right) x-B U\left(\tau_{j-1}\right) x\right\| .
\end{aligned}
$$

If $i(k-1)+1 \leq j \leq i(k)$ then $\tau_{j} \geq k-1$, so that the above inequality shows that

$$
\begin{aligned}
& \sum_{l=1}^{N} e^{-\lambda t_{l}} \| B U\left(t_{l}\right) x-B U\left(t_{l-1}\right) x \| \\
& \leq \sum_{k=[a]+1}^{\infty} e^{-\lambda(k-1)} \sum_{j=i(k-1)+1}^{i(k)}\left\|B U\left(\tau_{j}\right) x-B U\left(\tau_{j-1}\right) x\right\| .
\end{aligned}
$$

By (b-iii) we have $\sum_{j=i(k-1)+1}^{i(k)}\left\|B U\left(\tau_{j}\right) x-B U\left(\tau_{j-1}\right) x\right\| \leq K\|x\|$ for $k=1$. Let $k \geq 2$. By property (U2) we find 


$$
\begin{aligned}
(U(s+\xi)-U(s))(U(t+h) x & -U(t) x) / h \\
& =\frac{1}{h} \int_{t+s+\xi}^{t+s+\xi+h} U(\sigma) x d \sigma-\frac{1}{h} \int_{t+s}^{t+s+h} U(\sigma) x d \sigma
\end{aligned}
$$

for $s, t, \xi, h \geq 0$. Applying this equality with $s=\tau_{j-1}-(k-1), \xi=\tau_{j}-\tau_{j-1}$ and $t=k-1$ we have by the closedness of $B$ and (b-ii),

$$
\begin{gathered}
\sum_{j=i(k-1)+1}^{i(k)}\left\|\frac{1}{h} \int_{k-1}^{k-1+h}\left(B U\left(\sigma+\tau_{j}-(k-1)\right) x-B U\left(\sigma+\tau_{j-1}-(k-1)\right) x\right) d \sigma\right\| \\
=\sum_{j=i(k-1)+1}^{i(k)} \|\left(B U\left(\tau_{j}-(k-1)\right)-B U\left(\tau_{j-1}-(k-1)\right)\right) \\
\times(U((k-1)+h) x-U(k-1) x) / h \| .
\end{gathered}
$$

We estimate the right-hand side by (b-iii) and (2.1), and let $h \downarrow 0$ in the resulting inequality. This yields $\sum_{j=i(k-1)+1}^{i(k)}\left\|B U\left(\tau_{j}\right) x-B U\left(\tau_{j-1}\right) x\right\| \leq$ $K M e^{\omega(k-1)}\|x\|$. The desired estimate is obtained by substituting the two estimates shown above into (2.2).

To prove (ii-2), let $\beta>\omega$. Since $U(0)=0$, the desired assertion is obtained by using (ii-1) with $a=0, b=t$ and $\lambda=\beta$.

3. Perturbation theorems of Miyadera type for locally Lipschitz continuous integrated semigroups. According to the idea of Miyadera [7], we first introduce the number $r$ defined by (3.2) below. This number is used to obtain a lower estimate for perturbing operators.

Let $A$ be the generator of a locally Lipschitz continuous integrated semigroup $\{U(t) ; t \geq 0\}$ on $X$ satisfying (2.1), and let $B \in \mathfrak{P}(A)$. Then there exists a sequence $\left\{B_{n}\right\}$ in $B(X)$ such that conditions (P1) and (P2) are satisfied.

Let $x \in X, \lambda>\omega$ and $R>0$. For each partition $P=\left\{0=t_{0}<t_{1}<\right.$ $\left.\ldots<t_{N}=R\right\}$, consider the number

$$
r_{\lambda, R}^{(n)}(x)=\liminf _{|P| \rightarrow 0} \sum_{l=1}^{N} e^{-\lambda t_{l}}\left\|B_{n} U\left(t_{l}\right) x-B_{n} U\left(t_{l-1}\right) x\right\|
$$

for $n=1,2, \ldots$, where $|P|=\max _{1 \leq l \leq N}\left(t_{l}-t_{l-1}\right)$, and define

$$
r_{\lambda}=\sup _{\|x\| \leq 1} r_{\lambda}(x)
$$

where $r_{\lambda}(x)=\sup _{n \geq 1} r_{\lambda}^{(n)}(x)$ and $r_{\lambda}^{(n)}(x)=\sup _{R>0} r_{\lambda, R}^{(n)}(x)$. By Lemma 2.2 we have $r_{\lambda, R}^{(n)}(x) \leq\left(K+M K e^{-(\lambda-\omega)}\left(1-e^{-(\lambda-\omega)}\right)^{-1}\right)\|x\|$ for $n=1,2, \ldots$, so that $r_{\lambda}$ is finite. Since $r_{\lambda}$ is a nonnegative, nonincreasing function in 
$\lambda \in(\omega, \infty)$, the limit

$$
r=\lim _{\lambda \rightarrow \infty} r_{\lambda}
$$

exists and $0 \leq r<\infty$.

Lemma 3.1. Let $n \geq 1, \lambda>\omega$ and $x \in X$. The Riemann-Stieltjes integral $\int_{0}^{\infty} e^{-\lambda t} d B_{n} U(t) x:=\lim _{R \rightarrow \infty} \int_{0}^{R} e^{-\lambda t} d B_{n} U(t) x$ exists, and

$$
B_{n}(\lambda I-A)^{-1} x=\int_{0}^{\infty} e^{-\lambda t} d B_{n} U(t) x .
$$

Proof. Let $n \geq 1, \lambda>\omega$ and $x \in X$. Since $B_{n} \in B(X)$ and $U(\cdot) x$ is locally Lipschitz continuous in $X$ on $[0, \infty)$, we see that $B_{n} U(\cdot) x:[a, b] \rightarrow X$ is of bounded variation for $0 \leq a<b<\infty$. It follows that

$$
\lim _{|P| \rightarrow 0} \sum_{l=1}^{N} e^{-\lambda t_{l}}\left(B_{n} U\left(t_{l}\right) x-B_{n} U\left(t_{l-1}\right) x\right)=\int_{a}^{b} e^{-\lambda t} d B_{n} U(t) x
$$

for $0 \leq a<b<\infty$, where $P=\left\{a=t_{0}<t_{1}<\ldots<t_{N}=b\right\}$ is a partition of $[a, b]$. By Lemma 2.2 we have

$$
\left\|\int_{a}^{b} e^{-\lambda t} d B_{n} U(t) x\right\| \leq M K e^{-(\lambda-\omega)[a]}\left(1-e^{-(\lambda-\omega)}\right)^{-1}\|x\| \rightarrow 0
$$

as $b>a \rightarrow \infty$. This shows that the integral $\int_{0}^{\infty} e^{-\lambda t} d B_{n} U(t) x$ exists.

To prove (3.3) we notice that

$$
\int_{0}^{R} e^{-\lambda t} d B_{n} U(t) x=e^{-\lambda R} B_{n} U(R) x+\int_{0}^{R} \lambda e^{-\lambda t} B_{n} U(t) x d t
$$

for $R>0$. The desired result is obtained by letting $R \rightarrow \infty$.

The main result of the present paper is the following theorem, which extends the result of Miyadera [7, Theorem 1].

THEOREM 3.2. Let $A$ be the generator of a locally Lipschitz continuous integrated semigroup on $X$ and let $B \in \mathfrak{P}(A)$. Then for each $\varepsilon$ with $|\varepsilon|<1 / r$, where $1 / r=\infty$ if $r=0, A+\varepsilon B$ is the generator of a locally Lipschitz continuous integrated semigroup on $X$.

Proof. Let $\varepsilon$ satisfy $|\varepsilon|<1 / r$. We first show that for each $n \geq 1, A+\varepsilon B_{n}$ is the generator of a locally Lipschitz continuous integrated semigroup on $X$. For this purpose, let $n \geq 1$ and inductively define a sequence $\left\{V_{n}^{(k)}\right\}_{k=1}^{\infty}$ in $\operatorname{Lip}_{\text {loc }}([0, \infty) ; B(X))$ by

$$
\left\{\begin{array}{l}
V_{n}^{(0)}(t)=U(t) \quad \text { for } t \geq 0 \\
V_{n}^{(k)}(t) x=\int_{0}^{t} V_{n}^{(k-1)}(t-s) d \varepsilon B_{n} U(s) x \quad \text { for } x \in X \text { and } t \geq 0 .
\end{array}\right.
$$


Since $B_{n} U(\cdot) x:[0, \infty) \rightarrow X$ is of bounded variation on every compact subinterval of $[0, \infty), V_{n}^{(k)}(t)$ is well defined. Clearly, $V_{n}^{(k)}(0)=0$ for $k=$ $1,2, \ldots$ We shall show inductively that $V_{n}^{(k)} \in \operatorname{Lip}_{\text {loc }}([0, \infty) ; B(X))$ for each $k \geq 1$. By the definition (3.2) of $r$, there exists $\beta(>\omega)$ such that $|\varepsilon| r_{\beta}<1$, where $\omega$ is a number satisfying (2.1). Let $x \in X$ and $t, h \geq 0$. By the definition of $V_{n}^{(k)}(t)$ we have

$$
\begin{aligned}
V_{n}^{(k)}(t+h) x-V_{n}^{(k)}(t) x & =\int_{t}^{t+h} V_{n}^{(k-1)}(t+h-s) d \varepsilon B_{n} U(s) x \\
& +\int_{0}^{t}\left(V_{n}^{(k-1)}(t+h-s)-V_{n}^{(k-1)}(t-s)\right) d \varepsilon B_{n} U(s) x .
\end{aligned}
$$

If $\left\|V_{n}^{(k-1)}(\tau+\delta)-V_{n}^{(k-1)}(\tau)\right\| \leq M e^{\beta(\tau+\delta)} \delta\left(|\varepsilon| r_{\beta}\right)^{k-1}$ for $\tau, \delta \geq 0$, then

$$
\begin{aligned}
& \left\|\int_{t}^{t+h} V_{n}^{(k-1)}(t+h-s) d \varepsilon B_{n} U(s) x\right\| \\
& \quad \leq M e^{\beta(t+h)} h\left(|\varepsilon| r_{\beta}\right)^{k-1}|\varepsilon| \liminf _{|P| \rightarrow 0} \sum_{l=1}^{N} e^{-\beta t_{l}}\left\|B_{n} U\left(t_{l}\right) x-B_{n} U\left(t_{l-1}\right) x\right\|
\end{aligned}
$$

and

$$
\begin{aligned}
& \left\|\int_{0}^{t}\left(V_{n}^{(k-1)}(t+h-s)-V_{n}^{(k-1)}(t-s)\right) d \varepsilon B_{n} U(s) x\right\| \\
& \quad \leq \liminf _{|Q| \rightarrow 0} \sum_{l=1}^{\widehat{N}} M e^{\beta\left(t+h-s_{l}\right)} h\left(|\varepsilon| r_{\beta}\right)^{k-1}|\varepsilon|\left\|B_{n} U\left(s_{l}\right) x-B_{n} U\left(s_{l-1}\right) x\right\|,
\end{aligned}
$$

where $P$ and $Q$ are partitions of $[t, t+h]$ and $[0, t]$ respectively. Since $P \cup Q$ is a partition of $[0, t+h]$, we find by $(3.1)$ that

$$
\left\|V_{n}^{(k)}(t+h) x-V_{n}^{(k)}(t) x\right\| \leq M e^{\beta(t+h)} h\left(|\varepsilon| r_{\beta}\right)^{k-1}|\varepsilon| r_{\beta, t+h}^{(n)}(x),
$$

and inductively, $\left\|V_{n}^{(k)}(t+h)-V_{n}^{(k)}(t)\right\| \leq M e^{\beta(t+h)} h\left(|\varepsilon| r_{\beta}\right)^{k}$ for $t, h \geq 0$ and $k=1,2, \ldots$

Now, define a family $\left\{V_{n}(t) ; t \geq 0\right\}$ in $B(X)$ by $V_{n}(t) x=\sum_{k=0}^{\infty} V_{n}^{(k)}(t) x$ for $x \in X$ and $t \geq 0$, with the help of the estimate shown above. Clearly,

$$
\left\|V_{n}(t+h)-V_{n}(t)\right\| \leq M e^{\beta(t+h)} h\left(1-|\varepsilon| r_{\beta}\right)^{-1}
$$

for all $t \geq 0$ and $h \geq 0$. Since $(d / d t) \int_{0}^{t} V_{n}^{(k-1)}(t-s) B_{n} U(s) x d s=$ $\int_{0}^{t} V_{n}^{(k-1)}(t-s) d B_{n} U(s) x$, we inductively show 


$$
\lambda \int_{0}^{\infty} e^{-\lambda t} V_{n}^{(k)}(t) x d t=(\lambda I-A)^{-1}\left(\varepsilon B_{n}(\lambda I-A)^{-1}\right)^{k} x
$$

for $x \in X$ and $\lambda>\beta$, by using (1.1) and the theorem about the Laplace transform of a convolution. By (3.3) together with the definition of $r_{\beta}$, we find

$$
\left\|\varepsilon B_{n}(\lambda I-A)^{-1}\right\| \leq|\varepsilon| r_{\beta}<1
$$

for $\lambda>\beta$. Here we have used the fact that $r_{\lambda}$ is nonincreasing in $\lambda$. It follows that $\int_{0}^{\infty} \lambda e^{-\lambda t} V_{n}(t) x d t=(\lambda I-A)^{-1}\left(I-\varepsilon B_{n}(\lambda I-A)^{-1}\right)^{-1} x=$ $\left(\lambda I-\left(A+\varepsilon B_{n}\right)\right)^{-1} x$ for $x \in X$ and $\lambda>\beta$. By [1, Theorem 3.1] we see that $\left\{V_{n}(t) ; t \geq 0\right\}$ is an integrated semigroup on $X$, and its generator is $A+\varepsilon B_{n}$.

The desired claim will be derived from a convergence theorem (Theorem 4.1 in Appendix) for locally Lipschitz continuous integrated semigroups as follows: By condition (P1), the estimate that $\left\|\varepsilon B(\lambda I-A)^{-1}\right\| \leq|\varepsilon| r_{\beta}$ for $\lambda>\beta$ follows from (3.5). This estimate implies that $(\lambda I-(A+\varepsilon B))^{-1}$ exists as an element of $B(X)$. Clearly, $\lim _{n \rightarrow \infty}\left(A+\varepsilon B_{n}\right) x=(A+\varepsilon B) x$ for $x \in D(A)$, by condition (P1). Notice that the family $\left\{V_{n}(t) ; t \geq 0\right\}$ satisfies (3.4). Then Theorem 4.1 asserts that $A+\varepsilon B$ is the generator of a locally Lipschitz continuous integrated semigroup on $X$.

Combining Proposition 2.1 and Theorem 3.2 we have the following two results.

Corollary 3.3 [7, Theorem 2]. Let $A$ be the generator of a semigroup of class $\left(C_{0}\right)$ on $X$. If a linear operator $B$ in $X$ satisfies conditions (a-i) and (a-ii), then there exists an $\varepsilon_{0}>0$ which is finite or $\infty$ such that for each $\varepsilon$ with $|\varepsilon|<\varepsilon_{0}, A+\varepsilon B$ is the generator of a semigroup of class $\left(C_{0}\right)$ on $X$.

Remark 3.1. (1) By Remark 2.1, Corollary 3.3 is an extension of the result of Phillips [4, Section 13.4, Corollary 1].

(2) Voigt [10] obtained a similar result under weaker assumptions. We do not know whether his result can be obtained from ours.

COROLlary 3.4. Let $A$ be the generator of a locally Lipschitz continuous integrated semigroup on $X$. If a closed linear operator $B$ in $X$ satisfies conditions (b-i) through (b-iii), then there exists an $\varepsilon_{0}>0$ which is finite or $\infty$ such that for each $\varepsilon$ with $|\varepsilon|<\varepsilon_{0}, A+\varepsilon B$ is the generator of a locally Lipschitz continuous integrated semigroup on $X$.

The following result is a special case of Corollary 3.4.

Corollary 3.5 [5, Proposition 3.3]. If $A$ is the generator of a locally Lipschitz continuous integrated semigroup on $X$ and $B \in B(\overline{D(A)}, X)$ then 
$A+B$ is the generator of a locally Lipschitz continuous integrated semigroup on $X$.

Proof. It suffices to show that the number $r$ defined by (3.2) is zero. Let $M \geq 1$ and $\omega \geq 0$ be constants satisfying (2.1). As was shown in Proposition 2.1, the operator $B$ is an element of $\mathfrak{P}(A)$ by considering a sequence $\left\{B_{n}\right\}$ in $B(X)$ defined by $B_{n}=B(I-(1 / n) A)^{-1}$ for $n \geq n_{0}$, where $n_{0}=[\omega]+1$. Let $\lambda>\omega$ and $n \geq n_{0}$. If $P=\left\{0=t_{0}<t_{1}<\ldots<t_{N}=R\right\}$ is a partition of $[0, R]$ then by $(2.1)$ we have

$$
\left\|B_{n} U\left(t_{l}\right) x-B_{n} U\left(t_{l-1}\right) x\right\| \leq M_{B} M^{2} e^{\omega t_{l}}\left(t_{l}-t_{l-1}\right)(1-\omega / n)^{-1}\|x\|,
$$

where $M_{B}>0$ is a constant such that $\|B x\| \leq M_{B}\|x\|$ for $x \in \overline{D(A)}$. This estimate shows that $r_{\lambda, R}^{(n)}(x) \leq M_{B} M^{2}(1-\omega / n)^{-1}\left(\int_{0}^{R} e^{-(\lambda-\omega) t} d t\right)\|x\|$, which implies that $r=0$.

4. Appendix. This section is devoted to a convergence theorem for locally Lipschitz continuous integrated semigroups. Although such theorems may be known, we give the proof for completeness. It should be noticed that generalizations of the classical Trotter-Kato theorem are found in [2] and [6].

TheOREM 4.1. For each $n \geq 1$, let $A_{n}$ be the generator of a locally Lipschitz continuous integrated semigroup $\left\{V_{n}(t) ; t \geq 0\right\}$ on $X$ satisfying the condition

$$
\left\|V_{n}(t+h)-V_{n}(t)\right\| \leq M e^{\omega(t+h)} h \quad \text { for } t, h \geq 0,
$$

where $M \geq 1$ and $\omega \geq 0$ are independent of $n$. Assume that $A$ is a closed linear operator in $X$ satisfying the following two conditions:

(a) For each $x \in D(A)$, there exist $x_{n} \in D\left(A_{n}\right)$ such that $x_{n} \rightarrow x$ and $A_{n} x_{n} \rightarrow A x$ as $n \rightarrow \infty$.

(b) For some $\lambda_{0}>\omega$, the range $R\left(\lambda_{0} I-A\right)$ of $\lambda_{0} I-A$ is dense in $X$.

Then $A$ is the generator of a locally Lipschitz continuous integrated semigroup $\{V(t) ; t \geq 0\}$ on $X$. Moreover, for $x \in X$ we have

$$
\lim _{n \rightarrow \infty} V_{n}(t) x=V(t) x
$$

uniformly on every compact subinterval of $[0, \infty)$.

Proof. By (4.1) we have $(\omega, \infty) \subset \varrho\left(A_{n}\right)$ and $\left\|\left(\lambda I-A_{n}\right)^{-k}\right\| \leq$ $M(\lambda-\omega)^{-k}$ for $\lambda>\omega$ and $k=1,2, \ldots$ As in the proof of [8, Theorem 2.1] we see that $A$ is the generator of a locally Lipschitz continuous integrated semigroup $\{V(t) ; t \geq 0\}$ on $X$ and

$$
\lim _{n \rightarrow \infty}\left(\lambda I-A_{n}\right)^{-k} x=(\lambda I-A)^{-k} x
$$


for $x \in X, \lambda>\omega$ and $k=1,2, \ldots$ By [8, Lemma 2.2] we have

$$
\begin{aligned}
\left\|V(t) x-V_{n}(t) x\right\| \leq & 2 M(1-\lambda \omega)^{-[t / \lambda]} e^{\omega t}\left(([t / \lambda] \lambda-t)^{2}+[t / \lambda] \lambda^{2}\right)^{1 / 2}\|x\| \\
& +\sum_{l=1}^{[t / \lambda]} \lambda\left\|\left(I-\lambda A_{n}\right)^{-l} x-(I-\lambda A)^{-l} x\right\|
\end{aligned}
$$

for $n \geq 1, x \in X, t \geq 0$ and $\lambda>0$ with $\lambda \omega<1$. This estimate together with (4.3) implies (4.2).

\section{References}

[1] W. Arendt, Vector-valued Laplace transforms and Cauchy problems, Israel J. Math. 59 (1987), 327-352.

[2] W. Chojnacki, Multiplier algebras, Banach bundles, and one-parameter semigroups, Ann. Scuola Norm. Sup. Pisa Cl. Sci. 28 (1999), 287-322.

[3] G. Da Prato and E. Sinestrari, Differential operators with nondense domain, ibid. 14 (1987), 285-344.

[4] E. Hille and R. Phillips, Functional Analysis and Semi-Groups, Amer. Math. Soc. Colloq. Publ. 31, Amer. Math. Soc., Providence, RI, 1957.

[5] H. Kellermann and M. Hieber, Integrated semigroups, J. Funct. Anal. 84 (1989), 160-180.

[6] J. Kisyński, The Widder spaces, representations of the convolution algebra $L^{1}\left(\mathbb{R}^{+}\right)$ and one parameter semigroups of operators, preprint 588, Inst. Math., Polish Acad. Sci., 1998.

[7] I. Miyadera, On perturbation theory for semi-groups of operators, Tôhoku Math. J. 18 (1966), 299-310.

[8] N. Tanaka, Approximation of integrated semigroups by "integrated" discrete parameter semigroups, Semigroup Forum 55 (1997), 57-67.

[9] N. Tanaka and I. Miyadera, Exponentially bounded C-semigroups and integrated semigroups, Tokyo J. Math. 12 (1989), 99-115.

[10] J. Voigt, On the perturbation theory for strongly continuous semigroups, Math. Ann. 229 (1977), 163-171.

Department of Mathematics

Faculty of Science

Okayama University

Okayama 700-8530, Japan

E-mail: tanaka@math.okayama-u.ac.jp

Received May 17, 2002

Revised version November 18, 2002 